\title{
Structure-directed screening and analysis of thyroid-disrupting chemicals targeting transthyretin based on molecular recognition and chromatographic separation
}

Kai Huang, Xiu Wang, Hongxing Zhang, Lingshuai Zeng, Xiu Zhang, Bingmao Wang,

Yikai Zhou and Tao Jing*

State Key Laboratory of Environment Health (Incubation), Key Laboratory of

Environment and Health, Ministry of Education, Key Laboratory of Environment and

Health (Wuhan), Ministry of Environmental Protection, School of Public Health,

Tongji Medical College, Huazhong University of Science and Technology, \#13

Hangkong Road, Wuhan, Hubei, 430030, China.

* Corresponding Author. E-mail: jingtao@hust.edu.cn; telephone: +86(27)-83552611; fax: $+86(27)-83657765$.

Pages: 32

Figures: 10

Tables: 5 


\section{Table of Contents}



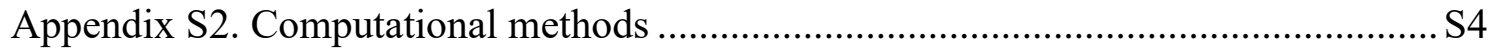

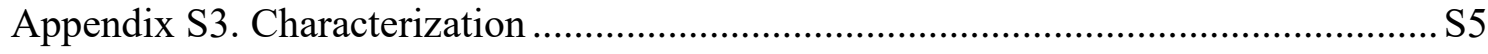

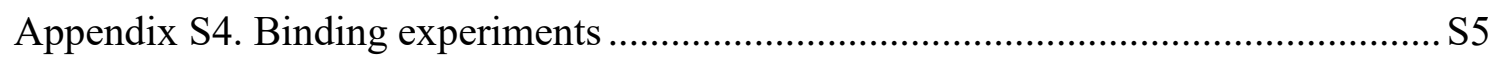

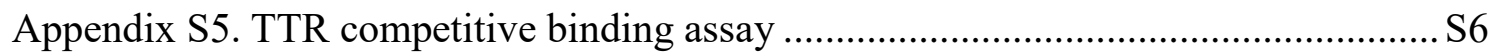

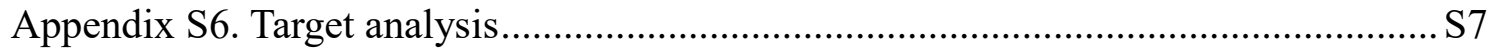

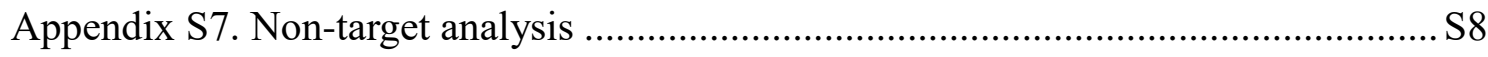

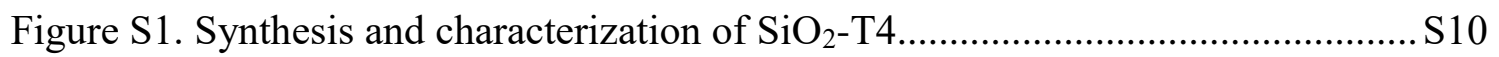

Figure S2. Box plot of retention times of twenty amino acids under different mobile

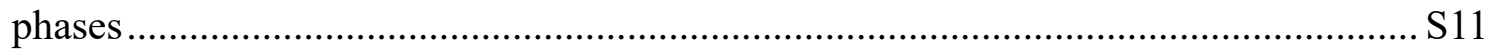

Figure S3. RDG analysis of the noncovalent interactions between IEMA and T4....... S12

Figure S4. Photos and fluorescence microscopic photographs of silica particles ........ S13

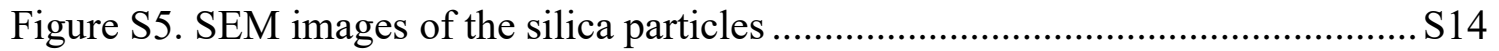

Figure S6. Binding performance of T4 on the imprinted composites..........................S15

Figure S7. Binding mechanisms of T4 with imprinted composites ......................... S16

Figure S8. Influence of the mobile phase on the retention of six model compounds... S17

Figure S9. Chromatograms of chemicals on $\mathrm{SiO}_{2}-\mathrm{TIP}-$ packed column ...................... S18

Figure S10. Concentrations of seven TTR-binding chemicals in dust samples

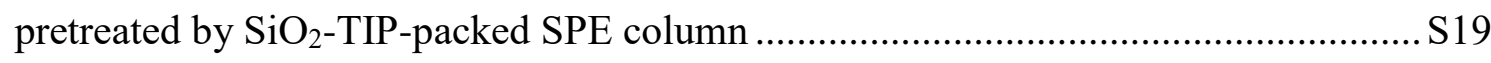

Table S1. Information of chemicals ................................................................. S2

Table S2. Retention factors, the reported activities, and RP values of chemicals in the 
Table S3. Parameters of isotherm and kinetic models for the adsorption of T4 on polymer coated particles S26

Table S4. Retention factors, predicted activities, $\mathrm{IC}_{50}$ values, and the tested activities of chemicals in the test set S27

Table S5. Recoveries of TTR-binding chemicals in dust samples and comparison with different SPE cartridges $\mathrm{S} 28$

References S29 


\section{Appendix S1. Synthesis of functional monomer}

The tryptophan-like monomer-2-(3-indol-yl)Ethylmethacrylamide (IEMA) was synthesized based on the reported method. ${ }^{1}$ Briefly, $10 \mathrm{mmol}$ of tryptamine was dissolved in $50 \mathrm{~mL}$ of tetrahydrofuran followed by adding $20 \mathrm{mmol}$ triethylamine. Then, $20 \mathrm{mmol}$ of methacryloyl chloride was added dropwise to this solution and the mixture was stirred for $12 \mathrm{~h}$ at room temperature. The supernatant was evaporated and then purified by column chromatography. ${ }^{1} \mathrm{H}$ NMR (400 MHz, deuterated DMSO): $10.81(\mathrm{~s}, 1 \mathrm{H}), 8.04(\mathrm{t}, \mathrm{J}=5.6 \mathrm{~Hz}, 1 \mathrm{H}), 7.55(\mathrm{~d}, \mathrm{~J}=7.8 \mathrm{~Hz}, 1 \mathrm{H}), 7.33(\mathrm{~d}, \mathrm{~J}=8.1 \mathrm{~Hz}$, 1H), $7.14(\mathrm{~d}, \mathrm{~J}=2.3 \mathrm{~Hz}, 1 \mathrm{H}), 7.06(\mathrm{t}, \mathrm{J}=7.0 \mathrm{~Hz}, 1 \mathrm{H}), 6.97(\mathrm{t}, \mathrm{J}=6.9 \mathrm{~Hz}, 1 \mathrm{H}), 5.64(\mathrm{~s}$, 1H), $5.34-5.29(\mathrm{~m}, 1 \mathrm{H}), 3.38(\mathrm{q}, \mathrm{J}=7.2 \mathrm{~Hz}, 2 \mathrm{H}), 2.85(\mathrm{t}, \mathrm{J}=7.9 \mathrm{~Hz}, 2 \mathrm{H}), 1.85(\mathrm{~s}$, 3H). MS (ESI): m/z calcd for $\mathrm{C} 14 \mathrm{H} 15 \mathrm{~N} 2 \mathrm{O}^{-}: 227.1$ [M-H]'; found: 277.1.

\section{Appendix S2. Computational methods}

Calculations were performed using the Gaussian16 suite of programs. Hybrid meta M06-2X exchange-correlation functional combined with the def2-SVP basis set were employed for geometry optimizations of the molecules. ${ }^{2,3}$ Solvent effect was considered by employing SMD model. ${ }^{4}$ The interaction energy $(\triangle \mathrm{E})(\mathrm{Eq} .(1))$ was calculated at M06-2X/def2-SVP level with the correction by the basis set superposition error method.

$$
\Delta E=E_{\text {complex }}-E_{\text {template }}-E_{\text {monomer }}
$$

Reduced density gradient (RDG) analysis was used to study the noncovalent interactions by Multiwfn program. ${ }^{5,6}$ The visualization of molecules and the gradient 
isosurface were carried out by Visual Molecular Dynamics (VMD). ${ }^{7}$ In a $\operatorname{sign}\left(\lambda_{2}\right) \rho$ color-filled RDG isosurface plot, blue color with negative $\operatorname{sign}\left(\lambda_{2}\right) \rho$ represented attractive interaction, green represented weak attraction and red with positive $\operatorname{sign}\left(\lambda_{2}\right) \rho$ represented repulsive interaction.

\section{Appendix S3. Characterization}

${ }^{1} \mathrm{H}$ NMR data of the synthesized monomer were recorded on a $400 \mathrm{MHz}$ Bruker AV-400 spectrometer (Germany). The synthesized particles were observed by an inverted Olympus IX 71 microscope (Japan) under blue light excitation. The morphologies were characterized using scanning electron microscopy (SEM) (Sirion200, FEI, Holland). The Fourier transform infrared (FTIR) spectra of materials were recorded using a Bruker VERTEX 70 spectrometer (Germany) with KBr pellet technique. X-ray photoelectron spectroscopy (XPS) was performed by Kratos AXIS Ultra DLD (Japan). Thermogravimetry-differential thermal analysis (Diamond TG-DTA, PerkinElmer) was performed by Diamond TG/DTA instrument (PerkinElmer, USA) at a heating rate of $10{ }^{\circ} \mathrm{C} \min ^{-1}$ in nitrogen atmosphere. Zeta-potentials were recorded by Delsa Nano analyzer (Beckman Coulter, USA).

\section{Appendix S4. Binding experiments}

The adsorption performances of $\mathrm{T} 4$ by the synthesized particles were studied by batch mode experiment. For the study of adsorption isotherms, $10 \mathrm{mg}$ of $\mathrm{SiO}_{2}$-TIP or $\mathrm{SiO}_{2}$-NIP were dispersed into $1 \mathrm{~mL}$ methanol solution with initial $\mathrm{T} 4$ concentrations 
of $50,100,200,300,500,700$ and $1000 \mu \mathrm{M}$. The mixture was shaken for $12 \mathrm{~h}$ at $25{ }^{\circ} \mathrm{C}$. The concentration of $\mathrm{T} 4$ in the supernatant was determined by UV-vis spectrometry (UV-1800, Shimadzu, Japan) at a wavelength of $300 \mathrm{~nm}$. The adsorption capacity was calculated by subtracting the supernatant $\mathrm{T} 4$ amount from the initial amount. Imprinting factor (IF) was calculated as the ratio between the amounts of T4 bound by the $\mathrm{SiO}_{2}$-TIP and the $\mathrm{SiO}_{2}$-NIP. Adsorption isotherms were studied using Freundlich and Langmuir models. For kinetic studies, $10 \mathrm{mg}$ sorbents were dispersed into $1 \mathrm{~mL}$ methanol solution at an initial T4 concentration of $100 \mu \mathrm{M}$. Adsorption capacities were obtained with different time intervals $(2,5,10,20,30,60,90,120$ and $150 \mathrm{~min}$ ). Pseudo-Frist order and second order kinetic models were applied for fitting kinetic data. Selective adsorption experiments were performed as that of isotherm study, by changing T4 with its analogues (T3, T2, DIT and Tyr) at the concentration of $10 \mu \mathrm{M}$. All the batch experiments were repeated for three times.

\section{Appendix S5. TTR competitive binding assay}

Competitive binding assay was carried out to investigate the affinity of chemicals with TTR. A fluorescence probe FITC-T4 was synthesized by the reported method. ${ }^{8}$ Miniaturized assays were conducted in 96-well polypropylene microplates according to the reported method. ${ }^{9}$ Briefly, $40 \mu \mathrm{L}$ of TTR $(250 \mathrm{nM})$ and $50 \mu \mathrm{L}$ of FITC-T4 (100 $\mathrm{nM}$ ) were mixed and incubated for $5 \mathrm{~min}$ at room temperature. Then, $10 \mu \mathrm{L}$ of testing chemicals in different concentrations ( $1 \mathrm{nM}$ to $1 \mathrm{mM})$ was added and incubated for 5 min. The fluorescence intensity was measured at $485 \mathrm{~nm}$ for the excitation 
wavelength and $528 \mathrm{~nm}$ for the emission wavelength using a Syngene multimode plate reader (BioTck, MA, USA). The $\mathrm{IC}_{50}$ value, which was the concentration of an inhibitor that was required for $50 \%$ inhibition, was obtained by fitting the dose-responsive curve. Relatively potency (RP) was defined as the ratio between the $\mathrm{IC}_{50}$ of $\mathrm{T} 4$ and the $\mathrm{IC}_{50}$ of the compound. The $\mathrm{IC}_{50}$ value of $\mathrm{T} 4(0.25 \mu \mathrm{M})$ was close to that of the reported method $(0.26 \mu \mathrm{M})$.

\section{Appendix S6. Target analysis}

A total of 10 indoor dust samples were collected from Wuhan, Hubei province in China. $100 \mathrm{mg}$ of the dust were vortexed with $5 \mathrm{~mL}$ of methanol for $30 \mathrm{~min}$ twice and the combined extracts were evaporated to $500 \mu \mathrm{L}$ under a gentle stream of nitrogen. For targeted analysis, the extraction solvent was further purified by on-line solid-phase extraction (SPE) using a $\mathrm{SiO}_{2}$-TIP-packed SPE column $(2.1 \mathrm{~mm} \times 10$ $\mathrm{mm}$ ). Briefly, $500 \mu \mathrm{L}$ of the extraction solvent was loaded onto the column, and the column was washed with $\mathrm{MeOH}$ at a flow rate of $0.5 \mathrm{~mL} \mathrm{~min}-1$ for $2 \mathrm{~min}$. Then, the analytes were backflushed from the SPE column to the analytical column (ZORBAX Eclipse XDB C18, $2.1 \mathrm{~mm} \times 100 \mathrm{~mm}, 3.5 \mu \mathrm{m}$, Agilent, USA). Chemicals were analysed using a Waters Alliance e2695 HPLC system coupled to a Waters Xevo TQD triple quadrupole mass spectrometer with an electrospray ionization (ESI) ion source. The composition of the mobile phase was $2 \mathrm{mM}$ ammonium acetate in $\mathrm{H}_{2} \mathrm{O}(\mathrm{A})$ and $0.01 \% \mathrm{HAc}$ in $\mathrm{MeOH}(\mathrm{B})$ with a gradient elution program: $20 \% \mathrm{~B}$ increased linearly to $90 \%$ in $5 \mathrm{~min}$, then increased to $95 \%$ in $5 \mathrm{~min}$ and held for $3 \mathrm{~min}$, and finally 
returned to the initial composition in $1 \mathrm{~min}$ and held for another $3 \mathrm{~min}$.

\section{Appendix S7. Non-target analysis}

The Dust sample was extracted with methanol as described in target analysis and evaporated to $100 \mu \mathrm{L}$ for non-target analysis. The extract was injected into an HPLC system equipped with a $\mathrm{SiO}_{2}$-TIP-packed column $(4.6 \mathrm{~mm} \times 100 \mathrm{~mm})$ for separation. The eluate from 5 to 30 min was collected and concentrated to $200 \mu \mathrm{L}$.

For non-target screening, $100 \mu \mathrm{L}$ of eluate was analysed by electrospray ionization Fourier transform ion cyclotron resonance mass spectrometry (ESI-FTICR-MS) (Bruker Daltonics, USA). MS scans of m/z from 100 to 1000 in both negative and positive modes were collected. The raw data were filtered with a signal-to-noise ratio $(\mathrm{S} / \mathrm{N})>3$, and peaks in the procedure blank were removed. Peaks of exact mass were matched with the database on the U.S. EPA CompTox Chemistry Dashboard, and suspect chemicals with mass difference within 0.001 Da were picked. Furthermore, non-targeted screening was performed on a Bruker SCION-456GC gas chromatograph coupled to a Bruker SCION TQ mass spectrometer (Bruker Daltonics, USA). A DB-5MS capillary column $(30 \mathrm{~m} \times 0.25 \mathrm{~mm}$ i.d., $0.25 \mu \mathrm{m}$ phase thickness, Agilent Technologies, USA) was used for separation. The mass spectrometer was operated in full scan mode (m/z range 50-1000) using electron ionization (EI, $70 \mathrm{eV})$. The compounds were identified by library search using the NIST database with AMDIS (NIST 08). Chemicals identified by FTICR-MS and GC-MS were regarded as the suspect candidates of TTR-binding chemicals. Some suspect candidates were 
further tested by $\mathrm{SiO}_{2}$-TIP-packed column for verifications. TTR binding assays were also carried out to investigate the affinity of suspect candidates as described in Appendix S5. 
a
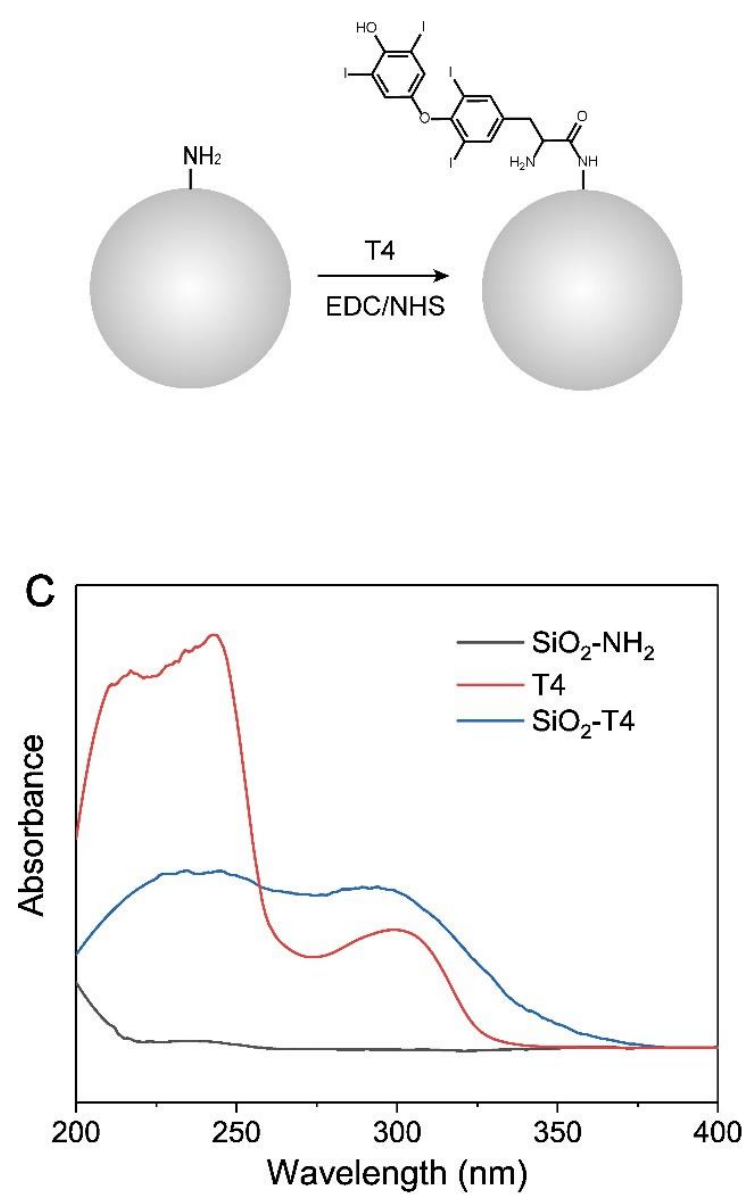
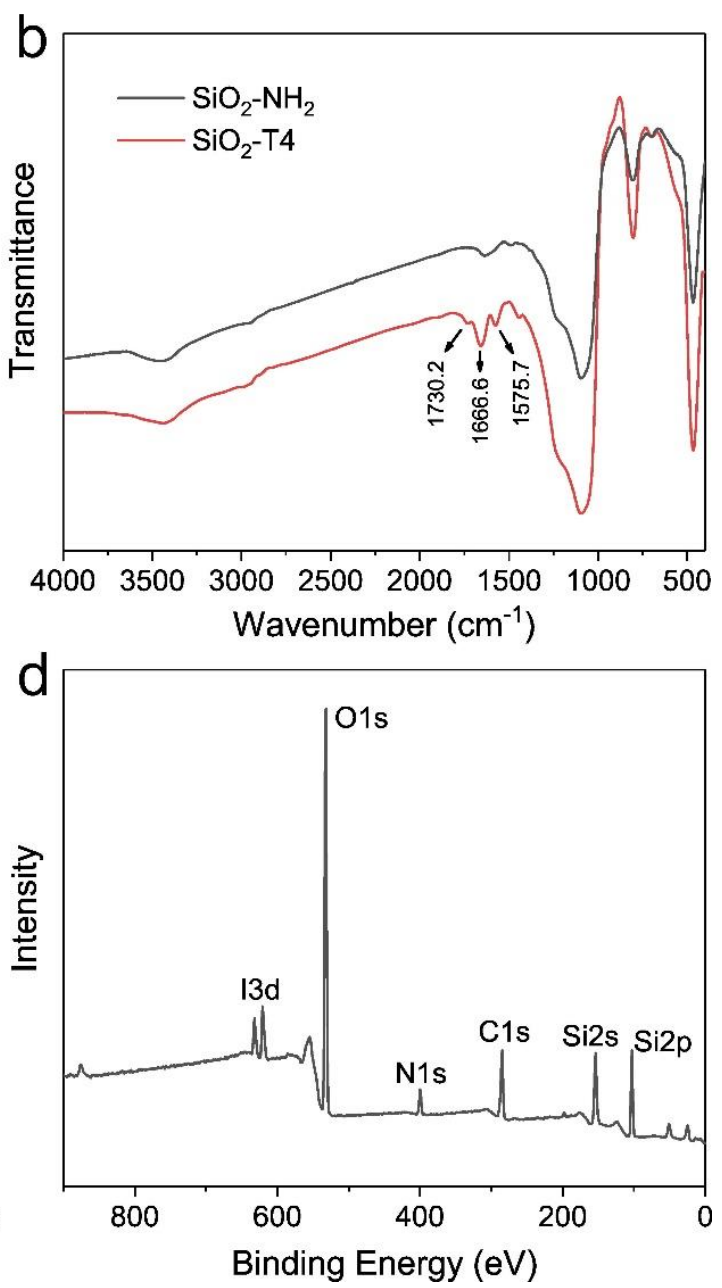

Figure $\mathrm{S} 1$. Synthesis and characterization of $\mathrm{SiO}_{2}-\mathrm{T} 4$. (a) $\mathrm{T} 4$ was grafted on $\mathrm{SiO}_{2}-\mathrm{NH}_{2}$ by EDC/NHS coupling reaction. (b) FT-IR spectra of $\mathrm{SiO}_{2}-\mathrm{NH}_{2}$ and $\mathrm{SiO}_{2}-\mathrm{T} 4$. The characteristic peaks at 1666.6 and $1575.7 \mathrm{~cm}^{-1}$ indicated the appearance of amide and the stretching of phenyl rings appeared at $1730.2 \mathrm{~cm}^{-1}$. (b) UV spectra of $\mathrm{SiO}_{2}-\mathrm{NH}_{2}$, $\mathrm{T} 4$ and $\mathrm{SiO}_{2}-\mathrm{T} 4$. The two characteristic peaks of $\mathrm{T} 4$ near 300 and $230 \mathrm{~nm}$ were observed on $\mathrm{SiO}_{2}$-T4. (c) XPS spectrum of $\mathrm{SiO}_{2}-\mathrm{T} 4$. The appearance of I3d peak indicated the successful modification of T4. 


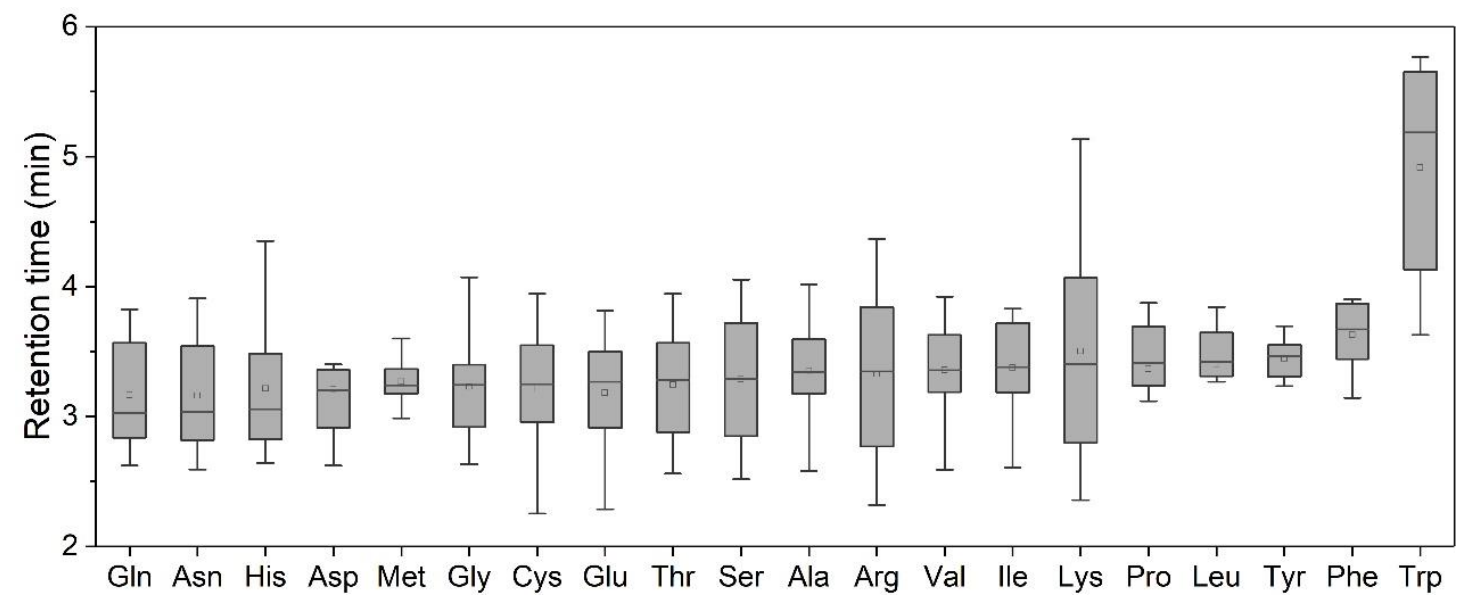

Figure S2. Box plot of retention times of twenty amino acids under different mobile phases. Mobile phase conditions: (1) $\mathrm{H}_{2} \mathrm{O}$; (2) $\mathrm{H}_{2} \mathrm{O} / \mathrm{ACN}=7 / 3$; (3) $\mathrm{H}_{2} \mathrm{O} / \mathrm{ACN}=5 / 5$; (4) $\mathrm{H}_{2} \mathrm{O} / \mathrm{ACN}=3 / 7$; (5) $10 \mathrm{mM}$ PBS (pH 3); (6) $10 \mathrm{mM}$ PBS (pH 5.6); (7) $10 \mathrm{mM}$ PBS (pH 7.4); (8) $10 \mathrm{mM}$ PBS (pH 8.9). The flow rate was $0.5 \mathrm{~mL} \mathrm{~min}^{-1}$ and wavelength of detector was $210 \mathrm{~nm}$. 


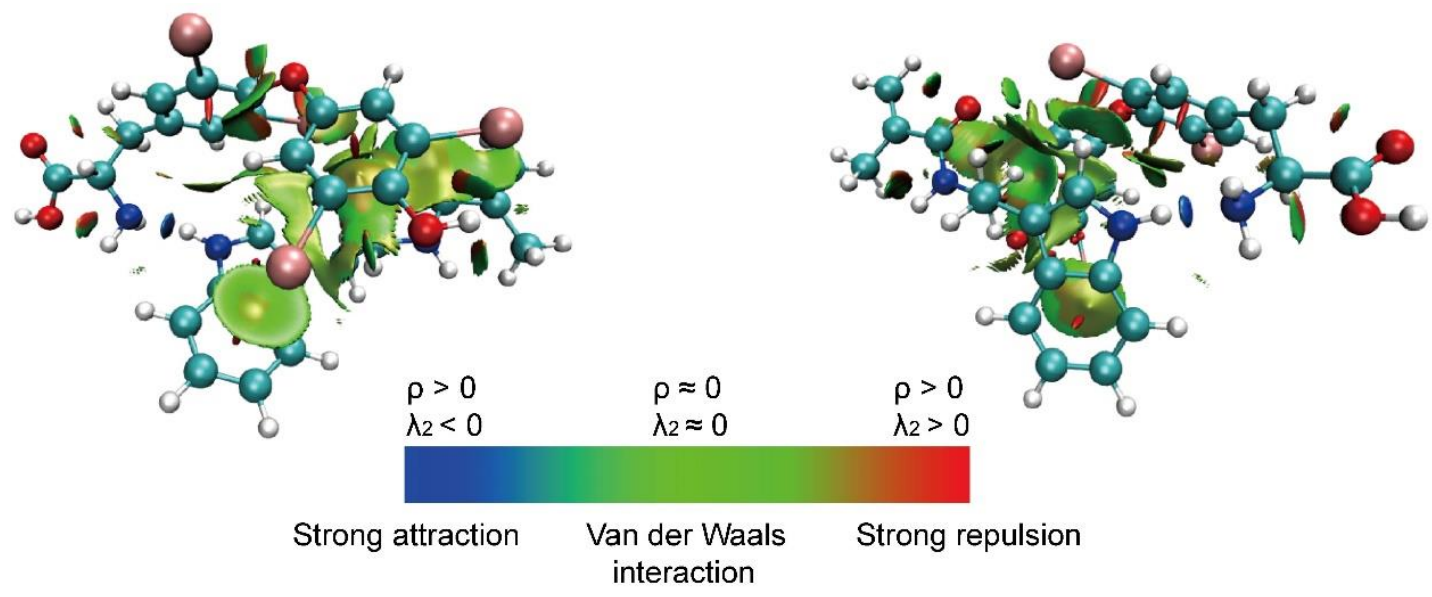

Figure S3. RDG analysis of the noncovalent interactions between IEMA and T4. 

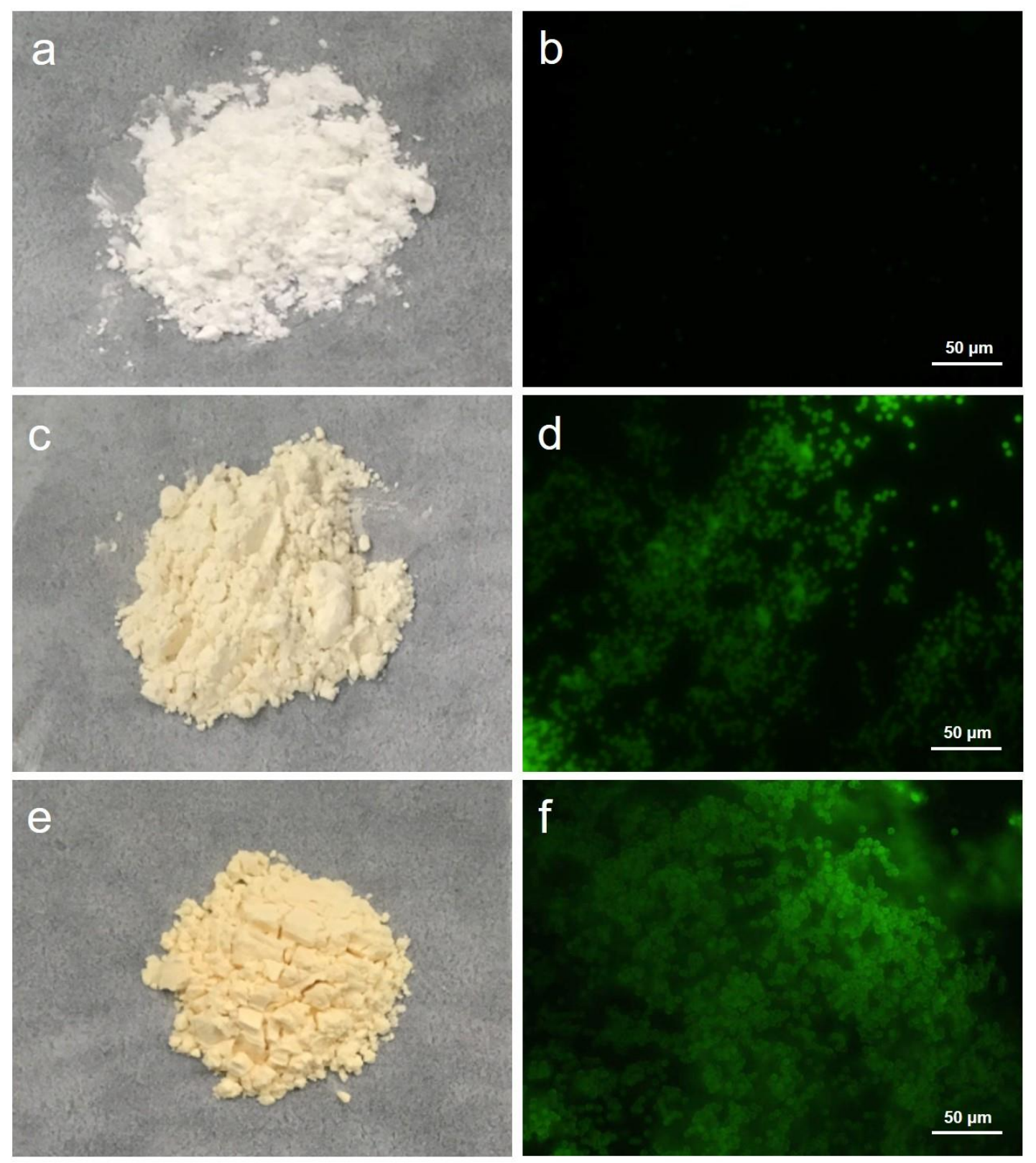

Figure S4. Photos and fluorescence microscopic photographs of silica particles. $(a, b)$ $\mathrm{SiO}_{2}-\mathrm{BIBB}$, (c, d) $\mathrm{SiO}_{2}-\mathrm{NIP}$, (e, f) $\mathrm{SiO}_{2}$-TIP. Fluorescence microscopy were conducted under blue light excitation. 

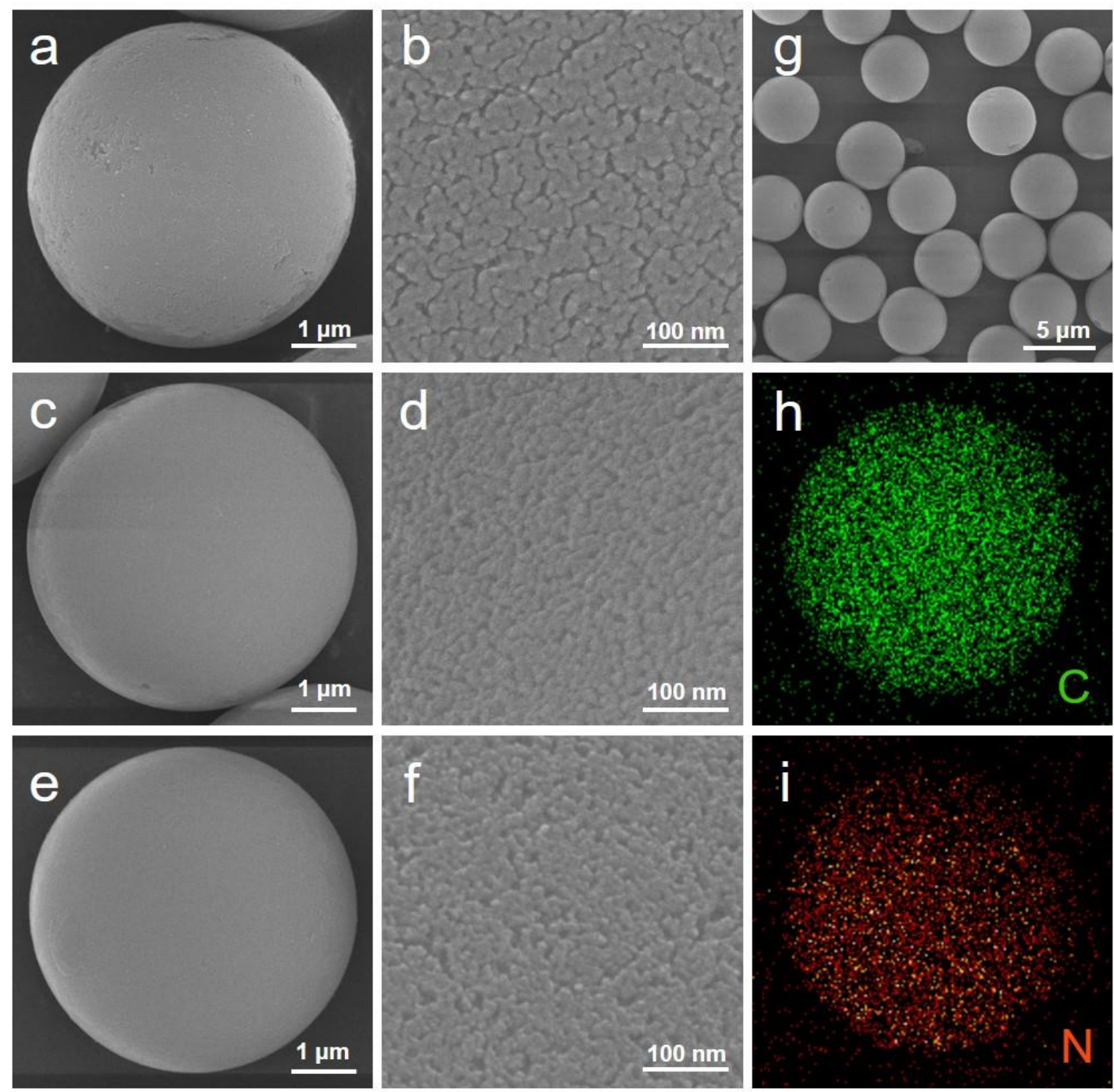

Figure S5. SEM images of the silica particles. $(a, b) \mathrm{SiO}_{2}-\mathrm{BIBB},(\mathrm{c}, \mathrm{d}) \mathrm{SiO}_{2}-\mathrm{NIP},(\mathrm{e}, \mathrm{f}$, g) $\mathrm{SiO}_{2}$-TIP. (h, i) EDX mapping images of $\mathrm{C}$ and $\mathrm{N}$ on $\mathrm{SiO}_{2}$-TIP. 

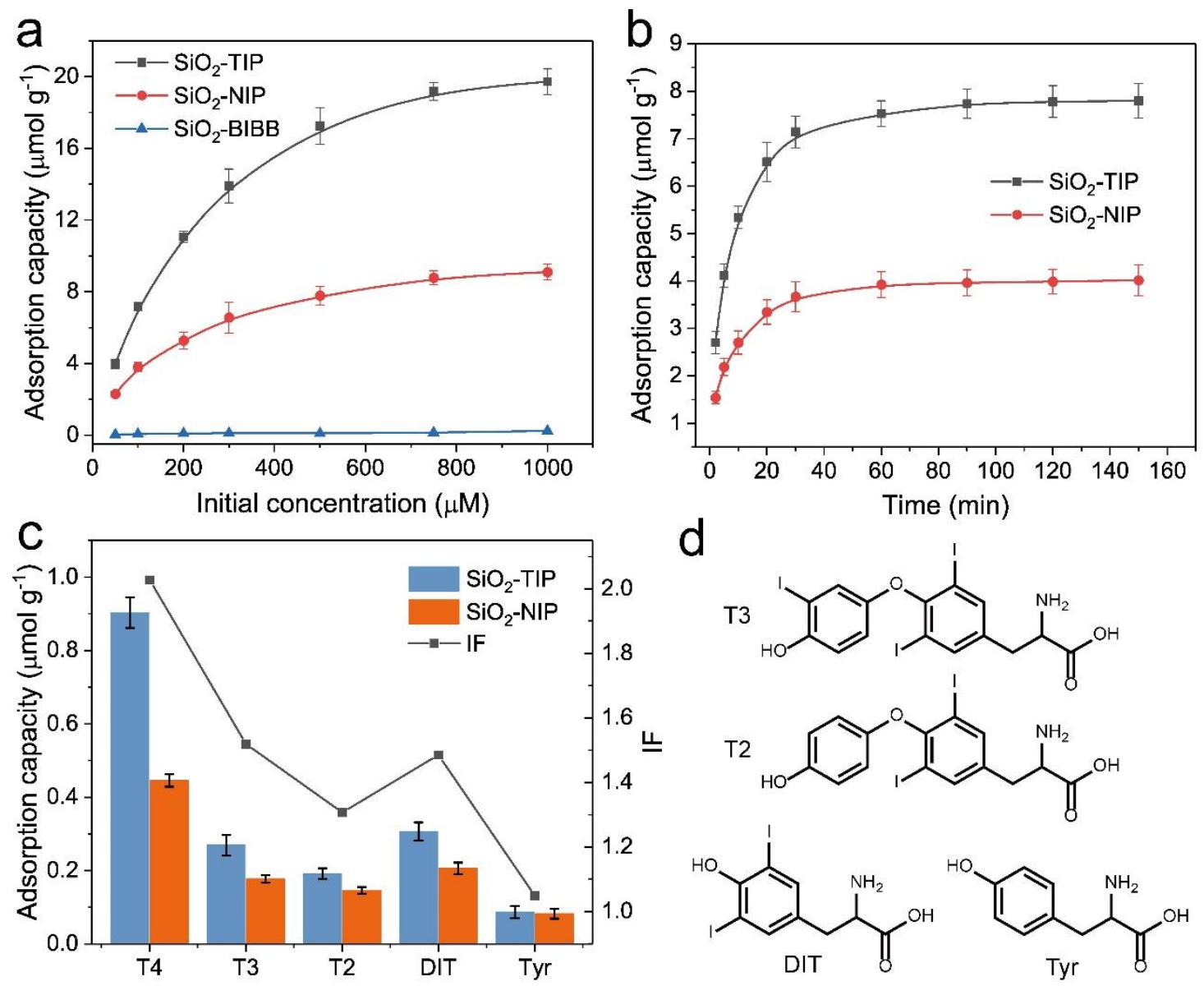

Figure S6. Binding performance of T4 on the imprinted composites. (a) Adsorption isotherms of T4 on modified silica particles. (b) Binding kinetic behaviors of T4 on polymer coated particles. Initial concentrations of T4 were $100 \mu \mathrm{M}$. (c) Adsorption behaviors of T4 analogues on polymer coated particles. Initial concentrations of T4 analogues were $10 \mu \mathrm{M}$. (d) Structures of T4 analogues. 

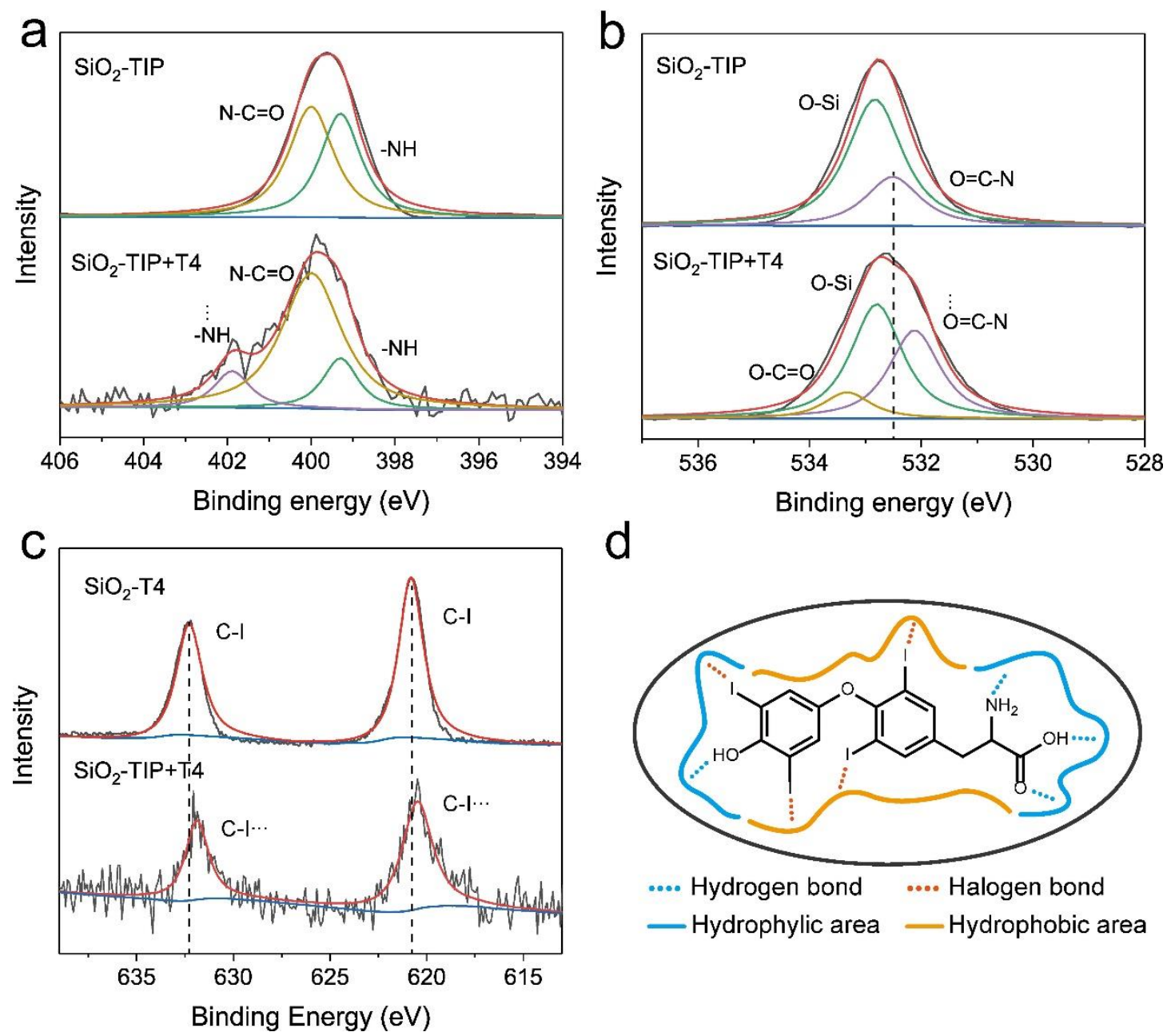

d



.... Hydrogen bond .... Halogen bond

- Hydrophylic area —Hydrophobic area

Figure S7. Binding mechanisms of T4 with imprinted composites. (a) High-resolution XPS spectra of $\mathrm{O} 1 \mathrm{~s}$ in $\mathrm{SiO}_{2}$-TIP before and after adsorption of T4. (b) High-resolution XPS spectra of I3d in $\mathrm{SiO}_{2}$-T4 and $\mathrm{SiO}_{2}$-TIP/T4 complex. (c) High-resolution XPS spectra of $\mathrm{N} 1 \mathrm{~s}$ in $\mathrm{SiO}_{2}$-TIP before and after adsorption of $\mathrm{T} 4$. (d) Illustration of binding mechanisms between T4 and imprinted composites. 

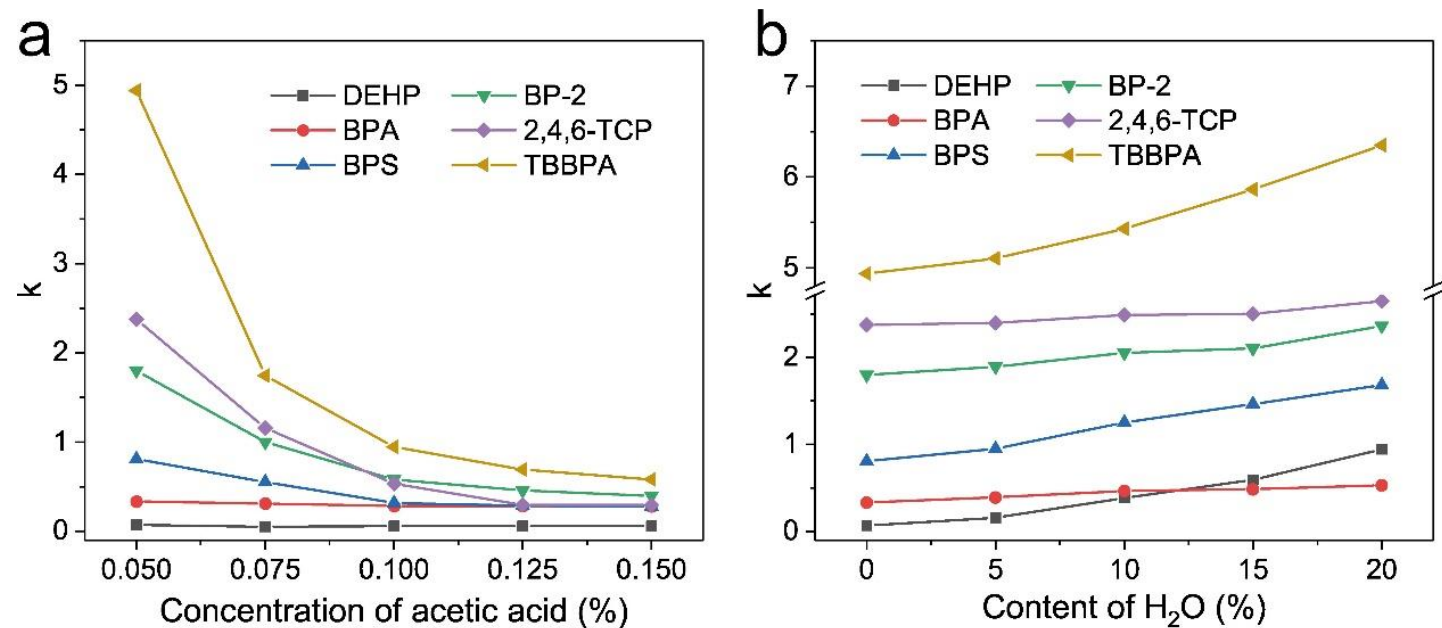

Figure S8. Influence of the mobile phase on the retention of six model compounds. (a) Retention factors of model compounds with different concentrations of HAc in $\mathrm{MeOH}$. (b) Retention factors of model compounds with different contents of $\mathrm{H}_{2} \mathrm{O}$ in $\mathrm{MeOH}(0.050 \% \mathrm{HAc})$. Flow rate: $0.5 \mathrm{~mL} \mathrm{~min}^{-1}$. 

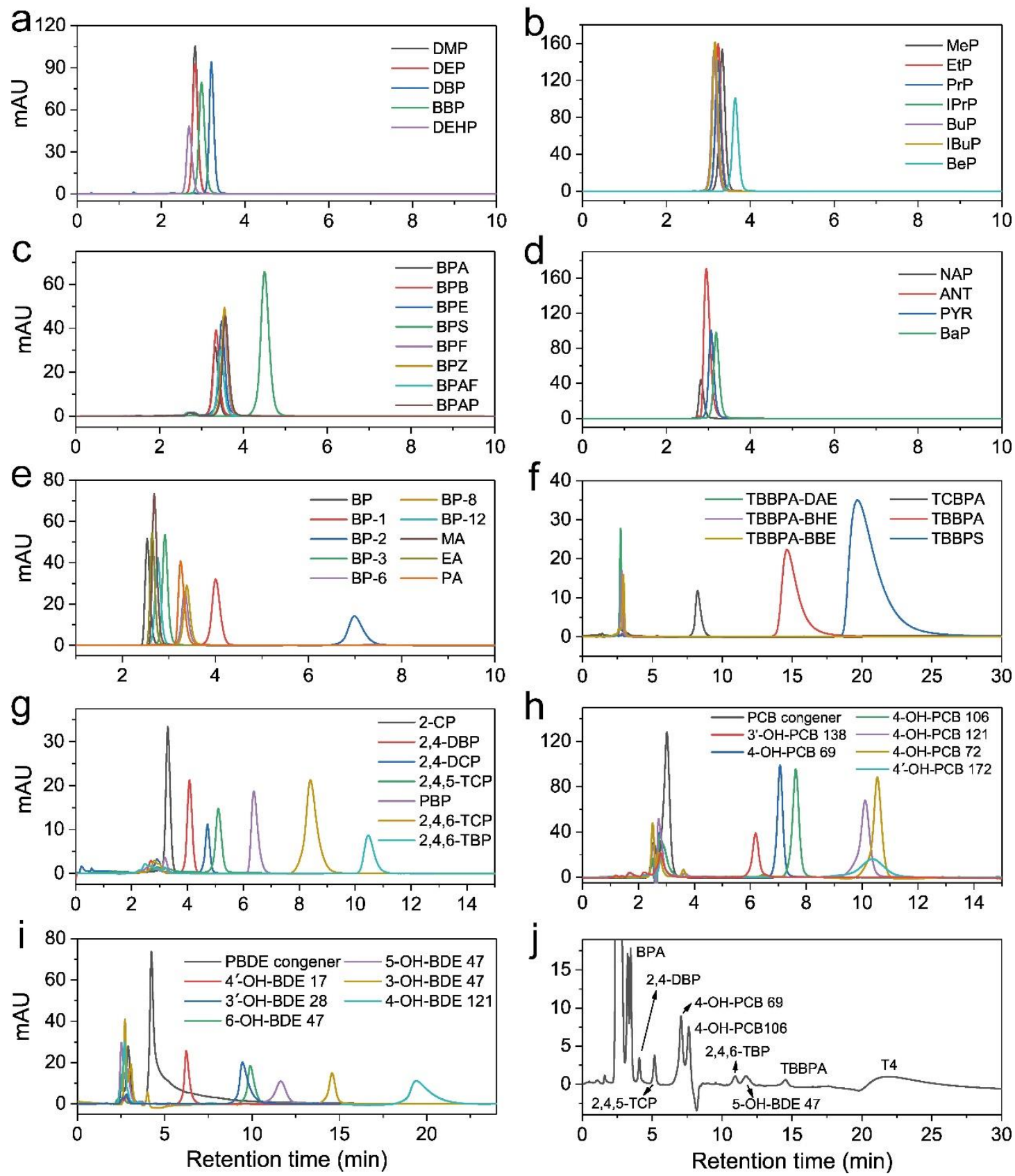

Figure S9. Chromatograms of chemicals on $\mathrm{SiO}_{2}$-TIP-packed column. (a) Phthalates. (b) Parabens. (c) Bisphenols. (d) PAHs. (e) UV filters. (f) Halogenated bisphenols. (g) Halogenated phenols. (h) PCBs and OH-PCBs. (i) PBDEs and OH-PBDEs. (j) Chemical mixtures. Mobile phase: $\mathrm{MeOH}(0.05 \% \mathrm{HAc})$. Flow rate: $0.5 \mathrm{~mL} \mathrm{~min}{ }^{-1}$. 


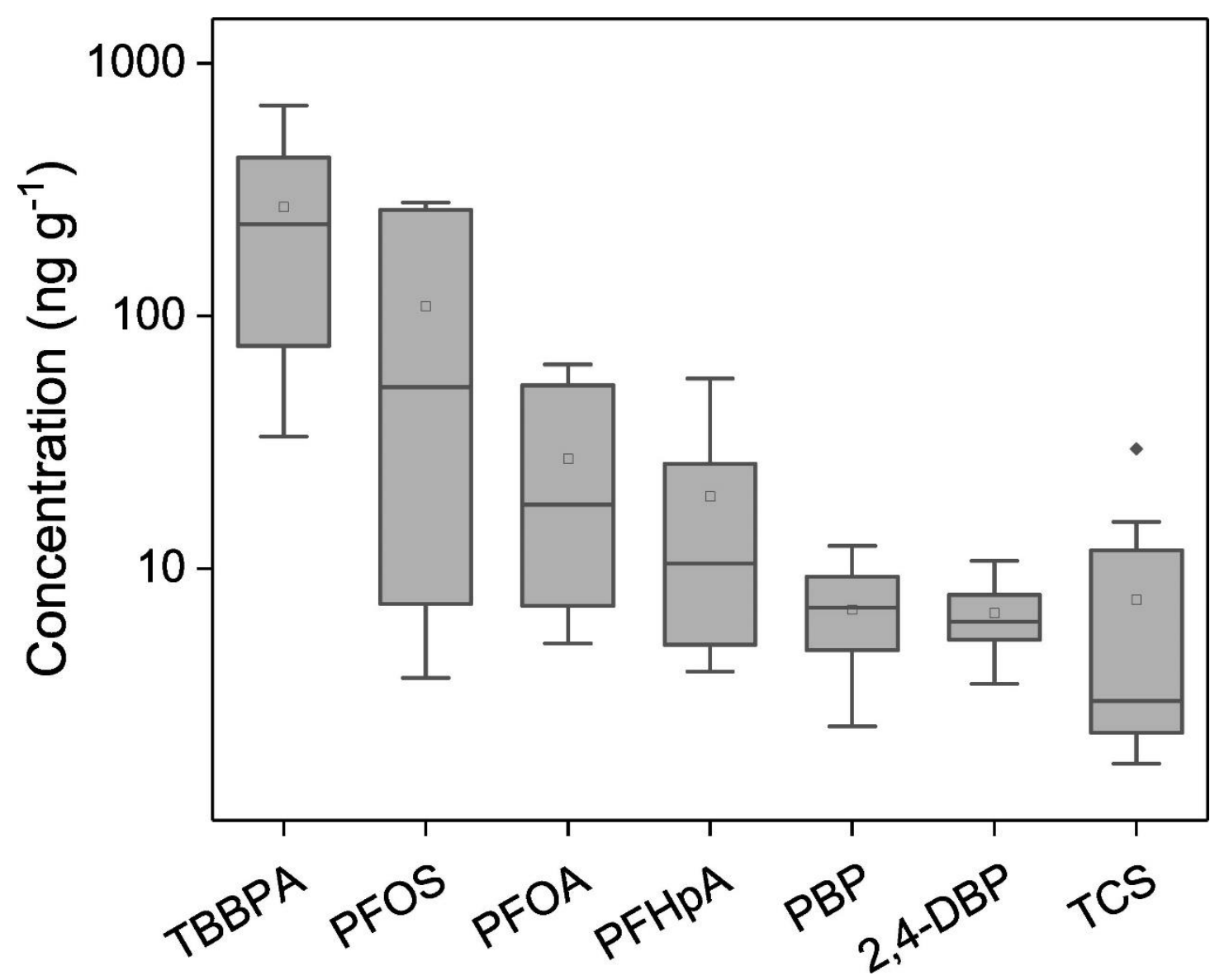

Figure S10. Concentrations of seven TTR-binding chemicals in dust samples pretreated by $\mathrm{SiO}_{2}$-TIP-packed SPE column. 
Table S1. Information of chemicals.

\begin{tabular}{|c|c|c|c|c|}
\hline Compound name & Abbreviation & Molecular formula & CAS No. & Purchased from \\
\hline \multicolumn{5}{|l|}{ Phthalates } \\
\hline Dimethyl phthalate & DMP & $\mathrm{C} 10 \mathrm{H} 10 \mathrm{O} 4$ & $131-11-3$ & Sigma-Aldrich \\
\hline Diethyl phthalate & DEP & $\mathrm{C} 12 \mathrm{H} 14 \mathrm{O} 4$ & $84-66-2$ & Sigma-Aldrich \\
\hline Butyl benzyl phthalate & BBP & $\mathrm{C} 19 \mathrm{H} 20 \mathrm{O} 4$ & $85-68-7$ & Sigma-Aldrich \\
\hline Bis(2-ethylhexyl) phthalate & DEHP & $\mathrm{C} 24 \mathrm{H} 38 \mathrm{O} 4$ & $117-81-7$ & Sigma-Aldrich \\
\hline Ethylparaben & EtP & $\mathrm{C} 9 \mathrm{H} 10 \mathrm{O} 3$ & $120-47-8$ & Sigma-Aldrich \\
\hline Propylparaben & $\operatorname{PrP}$ & $\mathrm{C} 10 \mathrm{H} 12 \mathrm{O} 3$ & $94-13-3$ & Sigma-Aldrich \\
\hline Isopropylparaben & IPrP & $\mathrm{C} 10 \mathrm{H} 12 \mathrm{O} 3$ & $4191-73-5$ & Sigma-Aldrich \\
\hline Butylparaben & $\mathrm{BuP}$ & $\mathrm{C} 11 \mathrm{H} 14 \mathrm{O} 3$ & $94-26-8$ & Sigma-Aldrich \\
\hline Isobutylparaben & $\mathrm{IBuP}$ & $\mathrm{C} 11 \mathrm{H} 14 \mathrm{O} 3$ & $4247-02-3$ & Sigma-Aldrich \\
\hline Benzylparaben & $\mathrm{BeP}$ & $\mathrm{C} 14 \mathrm{H} 12 \mathrm{O} 3$ & $94-18-8$ & Sigma-Aldrich \\
\hline Bisphenol E & $\mathrm{BPE}$ & $\mathrm{C} 14 \mathrm{H} 12 \mathrm{O} 2$ & $2081-08-5$ & TCI \\
\hline Bisphenol S & BPS & $\mathrm{C} 12 \mathrm{H} 10 \mathrm{O} 4 \mathrm{~S}$ & 80-09-1 & TCI \\
\hline Bisphenol F & $\mathrm{BPF}$ & $\mathrm{C} 13 \mathrm{H} 12 \mathrm{O} 2$ & $620-92-8$ & TCI \\
\hline Bisphenol Z & $\mathrm{BPZ}$ & $\mathrm{C} 18 \mathrm{H} 20 \mathrm{O} 2$ & $843-55-0$ & TCI \\
\hline Bisphenol AF & BPAF & C15H10F6O2 & $1478-61-1$ & TCI \\
\hline Bisphenol AP & BPAP & $\mathrm{C} 20 \mathrm{H} 18 \mathrm{O} 2$ & $1571-75-1$ & TCI \\
\hline Polycyclic aromatic hydroca & & & & \\
\hline
\end{tabular}




\begin{tabular}{|c|c|c|c|c|}
\hline Naphthalene & NAP & $\mathrm{C} 10 \mathrm{H} 8$ & $91-20-3$ & Aladdin \\
\hline Anthracene & ANT & $\mathrm{C} 14 \mathrm{H} 10$ & $120-12-7$ & Aladdin \\
\hline Pyrene & PYR & $\mathrm{C} 16 \mathrm{H} 10$ & $129-00-0$ & Aladdin \\
\hline Benzo(a)pyrene & $\mathrm{BaP}$ & $\mathrm{C} 20 \mathrm{H} 12$ & $50-32-8$ & Aladdin \\
\hline 2,4-Dihydroxybenzophenone & BP-1 & $\mathrm{C} 13 \mathrm{H} 10 \mathrm{O} 3$ & $131-56-6$ & Sigma-Aldrich \\
\hline 2,2',4,4'-Tetrahydroxybenzophenone & BP-2 & $\mathrm{C} 13 \mathrm{H} 10 \mathrm{O} 5$ & $131-55-5$ & Sigma-Aldrich \\
\hline Oxybenzone & BP-3 & $\mathrm{C} 14 \mathrm{H} 12 \mathrm{O} 3$ & $131-57-7$ & Sigma-Aldrich \\
\hline 2,2'-Dihydroxy-4-methoxybenzophenone & BP-8 & $\mathrm{C} 14 \mathrm{H} 12 \mathrm{O} 4$ & $131-53-3$ & Sigma-Aldrich \\
\hline Octabenzone & BP-12 & $\mathrm{C} 21 \mathrm{H} 26 \mathrm{O} 3$ & $1843-05-6$ & Sigma-Aldrich \\
\hline Benzophenone & $\mathrm{BP}$ & $\mathrm{C} 13 \mathrm{H} 10 \mathrm{O}$ & $119-61-9$ & Sigma-Aldrich \\
\hline Phenyl salicylate & $\mathrm{PA}$ & $\mathrm{C} 13 \mathrm{H} 10 \mathrm{O} 3$ & $118-55-8$ & Aladdin \\
\hline Methyl 4-aminobenzoate & MA & $\mathrm{C} 8 \mathrm{H} 9 \mathrm{NO} 2$ & $619-45-4$ & Aladdin \\
\hline Ethyl 4-aminobenzoate & EA & $\mathrm{C} 9 \mathrm{H} 11 \mathrm{NO} 2$ & 94-09-7 & Aladdin \\
\hline 1,1-dichloro-2,2-bis(4-chlorophenyl)ethene & 4,4'-DDE & $\mathrm{C} 14 \mathrm{H} 8 \mathrm{Cl} 4$ & $72-55-9$ & First Standard \\
\hline 2,4-Dichlorophenoxyacetic acid & $2,4-\mathrm{D}$ & $\mathrm{C} 8 \mathrm{H} 6 \mathrm{Cl} 2 \mathrm{O} 3$ & $94-75-7$ & First Standard \\
\hline \multicolumn{5}{|l|}{ Disinfectants } \\
\hline Triclosan & TCS & $\mathrm{C} 12 \mathrm{H} 7 \mathrm{Cl} 3 \mathrm{O} 2$ & $3380-34-5$ & Sigma-Aldrich \\
\hline Triclocarban & TCC & $\mathrm{C} 13 \mathrm{H} 9 \mathrm{Cl} 3 \mathrm{~N} 2 \mathrm{O}$ & $101-20-2$ & Sigma-Aldrich \\
\hline Hexachlorophene & $\mathrm{HCP}$ & $\mathrm{C} 13 \mathrm{H} 6 \mathrm{Cl} 6 \mathrm{O} 2$ & $70-30-4$ & Sigma-Aldrich \\
\hline \multicolumn{5}{|l|}{ Halogenated bisphenols } \\
\hline Tetrabromobisphenol A & TBBPA & $\mathrm{C} 15 \mathrm{H} 12 \mathrm{Br} 4 \mathrm{O} 2$ & 79-94-7 & TCI \\
\hline
\end{tabular}




\begin{tabular}{|c|c|c|c|c|}
\hline Tetrabromobisphenol S & TBBPS & $\mathrm{C} 12 \mathrm{H} 6 \mathrm{Br} 4 \mathrm{O} 4 \mathrm{~S}$ & $39635-79-5$ & Adamas \\
\hline Tetrachlorobisphenol A & TCBPA & $\mathrm{C} 15 \mathrm{H} 12 \mathrm{C} 14 \mathrm{O} 2$ & $79-95-8$ & TCI \\
\hline Tetrabromobisphenol A diallyl ether & TBBPA-DAE & $\mathrm{C} 21 \mathrm{H} 20 \mathrm{Br} 4 \mathrm{O} 2$ & $25327-89-3$ & TCI \\
\hline Tetrabromobisphenol A bis(2-hydroxyethyl) ether & TBBPA-BHE & $\mathrm{C} 19 \mathrm{H} 20 \mathrm{Br} 4 \mathrm{O} 4$ & $4162-45-2$ & TCI \\
\hline \multicolumn{5}{|l|}{ Alkylphenols } \\
\hline Octylphenol & $\mathrm{OP}$ & $\mathrm{C} 14 \mathrm{H} 22 \mathrm{O}$ & $140-66-9$ & Sigma-Aldrich \\
\hline Nonylphenol & NP & $\mathrm{C} 15 \mathrm{H} 24 \mathrm{O}$ & $25154-52-3$ & Sigma-Aldrich \\
\hline 2-Chlorophenol & $2-\mathrm{CP}$ & $\mathrm{C} 6 \mathrm{H} 5 \mathrm{ClO}$ & $95-57-8$ & Sigma-Aldrich \\
\hline 2,4-Dichlorophenol & 2,4-DCP & $\mathrm{C} 6 \mathrm{H} 4 \mathrm{Cl} 2 \mathrm{O}$ & $120-83-2$ & Sigma-Aldrich \\
\hline 2,4-Dibromophenol & 2,4-DBP & $\mathrm{C} 6 \mathrm{H} 4 \mathrm{Br} 2 \mathrm{O}$ & $615-58-7$ & Sigma-Aldrich \\
\hline 2,4,6-Trichlorophenol & 2,4,6-TCP & $\mathrm{C} 6 \mathrm{H} 3 \mathrm{Cl} 3 \mathrm{O}$ & $88-06-2$ & Sigma-Aldrich \\
\hline 2,4,5-Trichlorophenol & $2,4,5-\mathrm{TCP}$ & $\mathrm{C} 6 \mathrm{H} 3 \mathrm{Cl} 3 \mathrm{O}$ & $95-95-4$ & Sigma-Aldrich \\
\hline 2,4,6-Tribromophenol & 2,4,6-TBP & $\mathrm{C} 6 \mathrm{H} 3 \mathrm{Br} 3 \mathrm{O}$ & $118-79-6$ & Sigma-Aldrich \\
\hline 2,2',5,5'-Tetrachlorobiphenyl* & PCB 52 & $\mathrm{C} 12 \mathrm{H} 6 \mathrm{Cl} 4$ & $35693-99-3$ & \\
\hline $2,2^{\prime}, 4,5,5^{\prime}$-Pentachlorobiphenyl* & PCB 101 & $\mathrm{C} 12 \mathrm{H} 5 \mathrm{Cl} 5$ & $37680-73-2$ & \\
\hline 2,3',4,4',5-Pentachlorobiphenyl* & PCB 118 & $\mathrm{C} 12 \mathrm{H} 5 \mathrm{Cl} 5$ & $31508-00-6$ & Dr.Ehrenstorfer \\
\hline $2,2^{\prime}, 4,4^{\prime}, 5,5^{\prime}$-Hexachlorobiphenyl* & PCB 153 & $\mathrm{C} 12 \mathrm{H} 4 \mathrm{Cl} 6$ & $35065-27-1$ & \\
\hline 2,2',3,4,4',5'-Hexachlorobiphenyl* & PCB 138 & $\mathrm{C} 12 \mathrm{H} 4 \mathrm{Cl} 6$ & $35065-28-2$ & \\
\hline $2,2^{\prime}, 3,4,4^{\prime}, 5,5^{\prime}$-Heptachlorobiphenyl* & PCB 180 & $\mathrm{C} 12 \mathrm{H} 3 \mathrm{Cl} 7$ & $35065-29-3$ & \\
\hline 4-Hydroxy-2',3,4',6'-tetrachlorobiphenyl & 4-ОН-РСВ 69 & $\mathrm{C} 12 \mathrm{H} 6 \mathrm{Cl} 4 \mathrm{O}$ & $60233-24-1-4$ & AccuStandard \\
\hline 4-Hydroxy-2',3,5,5'-tetrachlorobiphenyl & 4-OH-РCB 72 & $\mathrm{C} 12 \mathrm{H} 6 \mathrm{Cl} 4 \mathrm{O}$ & $41464-42-0-4$ & AccuStandard \\
\hline
\end{tabular}




\begin{tabular}{|c|c|c|c|c|}
\hline 4-Hydroxy-2',3,3',4',5'-pentachlorobiphenyl & 4-OH-PCB 106 & $\mathrm{C} 12 \mathrm{H} 5 \mathrm{Cl} 5 \mathrm{O}$ & $67651-35-8$ & AccuStandard \\
\hline 4-Hydroxy-2',3,4',5,6'-pentachlorobiphenyl & 4-OH-PCB 121 & $\mathrm{C} 12 \mathrm{H} 5 \mathrm{Cl} 5 \mathrm{O}$ & $56558-18-0-4$ & AccuStandard \\
\hline 4'-Hydroxy-2,2',3,3',4,5,5'-heptachlorobiphenyl & 4'-OH-PCB 172 & $\mathrm{C} 12 \mathrm{H} 3 \mathrm{Cl} 7 \mathrm{O}$ & $158076-64-3$ & AccuStandard \\
\hline 3-Bromodiphenyl ether** & BDE 2 & $\mathrm{C} 12 \mathrm{H} 9 \mathrm{BrO}$ & $6876-00-2$ & \multirow{9}{*}{ BePure } \\
\hline 3,4-Dibromodiphenyl ether** & BDE 13 & $\mathrm{C} 12 \mathrm{H} 8 \mathrm{Br} 2 \mathrm{O}$ & $83694-71-7$ & \\
\hline $2,4,4^{\prime}$-Tribromodiphenyl ether** & BDE 28 & $\mathrm{C} 12 \mathrm{H} 7 \mathrm{Br} 3 \mathrm{O}$ & $41318-75-6$ & \\
\hline 2,2',3,4,4'-Pentabromodiphenyl ether** & BDE 85 & $\mathrm{C} 12 \mathrm{H} 5 \mathrm{Br} 5 \mathrm{O}$ & $182346-21-0$ & \\
\hline 2,2',3,4,4',5'-Hexabromodiphenyl ether** & BDE 138 & $\mathrm{C} 12 \mathrm{H} 4 \mathrm{Br} 6 \mathrm{O}$ & $182677-30-1$ & \\
\hline 2,3,3',4,4',5,6-Heptabromodiphenyl ether** & BDE 190 & $\mathrm{C} 12 \mathrm{H} 3 \mathrm{Br} 7 \mathrm{O}$ & $189084-68-2$ & \\
\hline 2,3,3',4,4',5,5',6-Octabromodiphenyl ether** & BDE 205 & $\mathrm{C} 12 \mathrm{H} 2 \mathrm{Br} 8 \mathrm{O}$ & $446255-56-7$ & \\
\hline $2,2^{\prime}, 3,3^{\prime}, 4^{\prime}, 5,5^{\prime}, 6$-Nonabromodiphenyl ether** & BDE 206 & $\mathrm{C} 12 \mathrm{HBr} 9 \mathrm{O}$ & $63387-28-0$ & \\
\hline Decabromodiphenyl ether** & BDE 209 & $\mathrm{C} 12 \mathrm{Br} 10 \mathrm{O}$ & $1163-19-5$ & \\
\hline 5-Hydroxy-2,2',4,4'-tetrabromodiphenyl ether & 5-OH-BDE 47 & $\mathrm{C} 12 \mathrm{H} 6 \mathrm{Br} 4 \mathrm{O} 2$ & $602326-30-7$ & AccuStandard \\
\hline 6-Hydroxy-2,2',4,4'-tetrabromodiphenyl ether & 6-OH-BDE 47 & $\mathrm{C} 12 \mathrm{H} 6 \mathrm{Br} 4 \mathrm{O} 2$ & $79755-43-4$ & AccuStandard \\
\hline 4'-Hydroxy-2,3',4,5',6-pentabromodiphenyl ether & 4-OH-BDE 121 & $\mathrm{C} 12 \mathrm{H} 5 \mathrm{Br} 5 \mathrm{O} 2$ & $91370-78-4$ & AccuStandard \\
\hline \multicolumn{5}{|l|}{ Natural compounds } \\
\hline Genistein & GE & $\mathrm{C} 15 \mathrm{H} 10 \mathrm{O} 5$ & $446-72-0$ & Sigma-Aldrich \\
\hline
\end{tabular}

\footnotetext{
*Mixed standard of 7 PCBs. **Mixed standard of 10 PBDEs.
} 
Table S2. Retention factors, the reported activities, and RP values of chemicals in the TTR dataset.

\begin{tabular}{|c|c|c|c|c|}
\hline No. & Compound & $\mathrm{k}$ & Activity & $\mathrm{RP}$ \\
\hline 1 & DBP & 0.283 & Non-binder & n.d. ${ }^{10}$ \\
\hline 2 & $\mathrm{BBP}$ & 0.189 & Non-binder & n.d. ${ }^{10}$ \\
\hline 3 & DEHP & 0.070 & Non-binder & n.d. ${ }^{10}$ \\
\hline 4 & $\mathrm{MeP}$ & 0.336 & Non-binder & n.d. ${ }^{11}$ \\
\hline 5 & EtP & 0.298 & Non-binder & $0.00053^{11}$ \\
\hline 6 & PrP & 0.293 & Non-binder & $0.0038^{11}$ \\
\hline 7 & $\mathrm{BuP}$ & 0.258 & Non-binder & $0.0017^{11}$ \\
\hline 8 & BPA & 0.334 & Non-binder & n.d. ${ }^{12-14}$ \\
\hline 9 & BPS & 0.811 & Non-binder & $0.002^{15}$ \\
\hline 10 & BP-2 & 1.801 & Weak binder & $0.18^{11}$ \\
\hline 11 & BP-6 & 0.340 & Non-binder & n.d. ${ }^{15}$ \\
\hline 12 & $4,4^{\prime}-\mathrm{DDT}$ & 0.430 & Non-binder & n.d. ${ }^{16}$ \\
\hline 13 & 4,4 '-DDE & 0.537 & Non-binder & n.d. ${ }^{16}$ \\
\hline 14 & $2,4-\mathrm{D}$ & 4.273 & Weak binder & $0.005^{10}$ \\
\hline 15 & TCS & 1.660 & Weak binder & $0.015^{10}$ \\
\hline 16 & TCC & 1.281 & Non-binder & n.d. ${ }^{10}$ \\
\hline 17 & $\mathrm{HCP}$ & 2.481 & Strong binder & $0.67^{17}$ \\
\hline 18 & TBBPA & 4.938 & Strong binder & $1^{12}$ \\
\hline 19 & TCBPA & 2.321 & Strong binder & $0.6^{12}$ \\
\hline 20 & TBBPA-BBE & 0.174 & Non-binder & n.d. ${ }^{14}$ \\
\hline 21 & $\mathrm{OP}$ & 0.168 & Non-binder & n.d. ${ }^{12}$ \\
\hline 22 & NP & 0.160 & Non-binder & n.d. ${ }^{18}$ \\
\hline 23 & 2-CP & 0.317 & Non-binder & $0.0004^{17}$ \\
\hline 24 & 2,4-DCP & 0.898 & Non-binder & n.d. ${ }^{12}$ \\
\hline 25 & 2,4-DBP & 0.631 & Weak binder & $0.06^{13}$ \\
\hline 26 & 2,4,6-TCP & 2.374 & Weak binder & $0.33^{17}$ \\
\hline 27 & $2,4,5-\mathrm{TCP}$ & 1.059 & Weak binder & $0.15^{17}$ \\
\hline 28 & 2,4,6-ТВP & 3.223 & Strong binder & $0.52^{9}$ \\
\hline 29 & PBP & 1.568 & Weak binder & $0.4^{19}$ \\
\hline 30 & PCB mixtures & 0.209 & Non-binder & n.d. ${ }^{10}$ \\
\hline 31 & 4-OH-PCB 69 & 1.831 & Strong binder & $0.6^{12}$ \\
\hline 32 & 4-OH-РCВ 72 & 3.234 & Strong binder & $2.5^{12}$ \\
\hline 33 & 4-OH-PCB 106 & 2.057 & Weak binder & $0.4^{12}$ \\
\hline 34 & 4-ОН-РCВ 121 & 3.065 & Strong binder & $0.8^{12}$ \\
\hline 35 & 4'-OH-PCB 172 & 3.150 & Strong binder & $3.8^{20}$ \\
\hline 36 & 5-OH-РCB 138 & 1.483 & Strong binder & $3.3^{20}$ \\
\hline 37 & PBDE mixtures & 0.699 & Non-binder & n.d. ${ }^{14,21}$ \\
\hline 38 & 4'-OH-BDE 17 & 1.501 & Strong binder & $1.5^{22}$ \\
\hline 39 & 3'-OH-BDE 28 & 2.792 & Strong binder & $0.7^{8}$ \\
\hline 40 & 3-OH-BDE 47 & 4.872 & Strong binder & $0.8^{12}$ \\
\hline 41 & 5-OH-BDE 47 & 3.671 & Weak binder & $0.4^{12}$ \\
\hline
\end{tabular}




\begin{tabular}{ccccc}
\hline 42 & 6-OH-BDE 47 & 2.970 & Weak binder & $0.2^{12}$ \\
43 & 4-OH-BDE 121 & 6.802 & Strong binder & $1.42^{13}$ \\
44 & GE & 3.356 & Weak binder & $0.4^{12}$ \\
45 & T4 & 7.794 & Strong binder & 1 \\
\hline
\end{tabular}

n.d. = not detected. 
Table S3. Parameters of isotherm and kinetic models for the adsorption of T4 on polymer coated particles.

\begin{tabular}{cccc}
\hline \multicolumn{1}{c}{ Models } & Parameters & $\mathrm{SiO}_{2}-\mathrm{TIP}$ & $\mathrm{SiO}_{2}-\mathrm{NIP}$ \\
\hline Isotherm models & & & \\
Langmuir model & $\mathrm{Q}_{\max }{ }^{*}\left(\mu \mathrm{mol} \mathrm{g}^{-1}\right)$ & 22.2 & 10.3 \\
& $\mathrm{~K}_{\mathrm{L}}\left({\left.\mathrm{L} \mu \mathrm{mol}^{-1}\right)}^{2}\right.$ & 0.008 & 0.006 \\
Freundlich model & $\mathrm{R}^{2}$ & 0.9947 & 0.9917 \\
& $\mathrm{~K}_{\mathrm{F}}\left(\mu \mathrm{mol} \mathrm{g}^{-1}\right)$ & 2.1 & 0.9 \\
Kinetic models & $\mathrm{n}$ & 2.905 & 2.842 \\
Pseudo-Frist order & $\mathrm{R}^{2}$ & 0.9585 & 0.9713 \\
& & & \\
Pseudo-Second order & $\mathrm{k}_{1}\left(\mathrm{~min}^{-1}\right)$ & 0.1221 & 2.9889 \\
& $\mathrm{R}^{2}$ & 0.9055 & 0.9115 \\
& $\mathrm{R}^{2}$ & 0.022 & 0.057 \\
\hline
\end{tabular}

* $\mathrm{Q}_{\max }$ denotes the maximum adsorption amount of T4. 
Table S4. Retention factors, predicted activities, $\mathrm{IC}_{50}$ values, and the tested activities of chemicals in the test set.

\begin{tabular}{|c|c|c|c|c|c|}
\hline $\begin{array}{c}\text { No } \\
\text {. }\end{array}$ & Compound & $\mathrm{k}$ & Predicted activity & $\begin{array}{l}\mathrm{IC}_{50} \\
(\mu \mathrm{M})\end{array}$ & Tested activity \\
\hline 1 & DMP & 0.127 & Non-binder & n.d. & Non-binder \\
\hline 2 & DEP & 0.126 & Non-binder & n.d. & Non-binder \\
\hline 3 & IPrP & 0.265 & Non-binder & n.d. & Non-binder \\
\hline 4 & $\mathrm{IBuP}$ & 0.267 & Non-binder & n.d. & Non-binder \\
\hline 5 & $\mathrm{BeP}$ & 0.460 & Non-binder & 5.43 & Binder \\
\hline 6 & BPB & 0.343 & Non-binder & 14.4 & Non-binder \\
\hline 7 & $\mathrm{BPE}$ & 0.397 & Non-binder & n.d. & Non-binder \\
\hline 8 & $\mathrm{BPF}$ & 0.419 & Non-binder & n.d. & Non-binder \\
\hline 9 & $\mathrm{BPZ}$ & 0.423 & Non-binder & n.d. & Non-binder \\
\hline 10 & BPAF & 0.386 & Non-binder & 25.9 & Non-binder \\
\hline 11 & BPAP & 0.431 & Non-binder & n.d. & Non-binder \\
\hline 12 & NAP & 0.149 & Non-binder & n.d. & Non-binder \\
\hline 13 & ANT & 0.185 & Non-binder & n.d. & Non-binder \\
\hline 14 & PYR & 0.230 & Non-binder & n.d. & Non-binder \\
\hline 15 & $\mathrm{BaP}$ & 0.303 & Non-binder & n.d. & Non-binder \\
\hline 16 & BP-1 & 0.606 & Non-binder & 2.53 & Binder \\
\hline 17 & BP-3 & 0.170 & Non-binder & 10.64 & Non-binder \\
\hline 18 & BP-8 & 0.357 & Non-binder & n.d. & Non-binder \\
\hline 19 & BP-12 & 0.107 & Non-binder & n.d. & Non-binder \\
\hline 20 & $\mathrm{BP}$ & 0.016 & Non-binder & n.d. & Non-binder \\
\hline 21 & PA & 0.308 & Non-binder & n.d. & Non-binder \\
\hline 22 & MA & 0.075 & Non-binder & n.d. & Non-binder \\
\hline 23 & EA & 0.067 & Non-binder & n.d. & Non-binder \\
\hline 24 & $\mathrm{HCH}$ & 0.325 & Non-binder & n.d. & Non-binder \\
\hline 25 & TBBPS & 6.890 & Binder & 0.63 & Binder \\
\hline 26 & TBBPA-DAE & 0.097 & Non-binder & n.d. & Non-binder \\
\hline 27 & TBBPA-BHE & 0.125 & Non-binder & n.d. & Non-binder \\
\hline
\end{tabular}

n.d. = not detected. 
Table S5. Recoveries of TTR-binding chemicals in dust samples and comparison with different SPE cartridges.

\begin{tabular}{|c|c|c|c|c|c|}
\hline Compounds & TIP & $\mathrm{MCX}^{20}$ & $\mathrm{MAX}^{23}$ & $\mathrm{MAX}^{24}$ & $\mathrm{HLB}^{25}$ \\
\hline TCS & $104.0 \pm 17.4$ & 110 & - & - & - \\
\hline 2,4-DCP & $87.8 \pm 14.8$ & - & - & - & - \\
\hline 2,4-DBP & $82.5 \pm 16.0$ & - & - & - & - \\
\hline 2,4,6-TCP & $87.9 \pm 10.5$ & - & - & - & - \\
\hline $2,4,5-\mathrm{TCP}$ & $105.0 \pm 16.4$ & 113 & - & - & - \\
\hline 2,4,6-TBP & $94.4 \pm 6.3$ & 110 & 74 & - & - \\
\hline PBP & $97.3 \pm 18.7$ & - & 89 & 70 & - \\
\hline TCBPA & $104.6 \pm 12.6$ & - & - & - & - \\
\hline TBBPA & $93.1 \pm 11.5$ & - & 75 & 95 & - \\
\hline TBBPS & $95.3 \pm 8.2$ & - & - & - & - \\
\hline 4'-OH-РCB 172 & $95.5 \pm 15.9$ & - & 59 & - & - \\
\hline 5-ОН-РCВ 138 & $101.9 \pm 17.5$ & 102 & 69 & - & - \\
\hline 4-OH-PCB 107 & $91.9 \pm 16.7$ & 105 & 88 & 90 & - \\
\hline 4-OH-PCB 72 & $85.9 \pm 5.4$ & - & - & - & - \\
\hline 4-OH-CB146 & $99.5 \pm 16.0$ & - & 81 & 78 & - \\
\hline 3-OH-CB153 & $98.8 \pm 17.4$ & - & 83 & - & - \\
\hline 4-OH-CB187 & $87.0 \pm 15.7$ & 71 & 70 & 91 & - \\
\hline 4'-OH-BDE 17 & $96.8 \pm 18.7$ & - & - & 72 & 63 \\
\hline 3'-OH-BDE 28 & $85.4 \pm 12.2$ & - & - & - & - \\
\hline 3-OH-BDE 47 & $89.6 \pm 6.9$ & 76 & - & - & 73 \\
\hline 5-OH-BDE 47 & $93.1 \pm 8.7$ & 102 & 78 & 99 & 101 \\
\hline 6-OH-BDE 47 & $105.5 \pm 10.3$ & - & 57 & 95 & 102 \\
\hline 4'-OH-BDE 49 & $89.7 \pm 14.9$ & 120 & - & 98 & 112 \\
\hline 4-OH-BDE 90 & $90.7 \pm 5.6$ & - & - & - & 63 \\
\hline 6'-OH-BDE 99 & $96.9 \pm 13.1$ & - & - & - & 107 \\
\hline 5'-OH-BDE 99 & $90.9 \pm 8.6$ & - & - & - & 113 \\
\hline 3-OH-BDE 154 & $97.8 \pm 18.5$ & - & - & - & - \\
\hline PFHxS & $93.6 \pm 11.4$ & 87 & - & - & - \\
\hline PFHpA & $115.8 \pm 8.9$ & 104 & - & - & - \\
\hline PFOA & $102.5 \pm 12.6$ & 83 & - & - & - \\
\hline PFOS & $93.7 \pm 7.4$ & 33 & - & - & - \\
\hline PFNA & $89.7 \pm 13.2$ & 51 & - & - & - \\
\hline PFDA & $92.9 \pm 17.8$ & 24 & - & - & - \\
\hline
\end{tabular}




\section{References}

(1) Ilgin, P.; Zorer, O. S.; Ozay, O.;Boran, G. Synthesis and characterization of 2-hydroxyethylmethacrylate/2-(3-indol-yl)ethylmethacrylamide-based novel hydrogels as drug carrier with in vitro antibacterial properties. J. Appl. Polym. Sci. 2017, 134, (47), 45550 .

(2) Zhao, Y.; Truhlar, D. G. The M06 suite of density functionals for main group thermochemistry, thermochemical kinetics, noncovalent interactions, excited states, and transition elements: two new functionals and systematic testing of four M06-class functionals and 12 other functionals. Theor. Chem. Acc. 2008, 120, (1), 215-241.

(3) Weigend, F.;Ahlrichs, R. Balanced basis sets of split valence, triple zeta valence and quadruple zeta valence quality for $\mathrm{H}$ to $\mathrm{Rn}$ : Design and assessment of accuracy. Phys. Chem. Chem. Phys. 2005, 7, (18), 3297-3305.

(4) Marenich, A. V.; Cramer, C. J.;Truhlar, D. G. Universal Solvation Model Based on Solute Electron Density and on a Continuum Model of the Solvent Defined by the Bulk Dielectric Constant and Atomic Surface Tensions. J. Phys. Chem. B 2009, 113, (18), 6378-6396.

(5) Johnson, E. R.; Keinan, S.; Mori-Sánchez, P.; Contreras-García, J.; Cohen, A. J.;Yang, W. Revealing Noncovalent Interactions. J. Am. Chem. Soc. 2010, 132, (18), 6498-6506.

(6) Lu, T.;Chen, F. Multiwfn: A multifunctional wavefunction analyzer. J. Comput. Chem. 2012, 33, (5), 580-592.

(7) Humphrey, W.; Dalke, A.;Schulten, K. VMD: Visual molecular dynamics. J. Mol. Graph. 1996, 14, (1), 33-38.

(8) Ren, X. M.;Guo, L. H. Assessment of the binding of hydroxylated polybrominated diphenyl ethers to thyroid hormone transport proteins using a site-specific fluorescence probe. Environ. Sci. Technol. 2012, 46, (8), 4633-4640.

(9) Ouyang, X.; Froment, J.; Leonards, P. E. G.; Christensen, G.; Tollefsen, K. E.; de Boer, J.; Thomas, K. V.;Lamoree, M. H. Miniaturization of a transthyretin binding assay using a fluorescent probe for high throughput screening of thyroid hormone 
disruption in environmental samples. Chemosphere 2017, 171, 722-728.

(10)Weiss, J. M.; Andersson, P. L.; Zhang, J.; Simon, E.; Leonards, P. E.; Hamers, T.;Lamoree, M. H. Tracing thyroid hormone-disrupting compounds: database compilation and structure-activity evaluation for an effect-directed analysis of sediment. Anal. Bioanal. Chem. 2015, 407, (19), 5625-5634.

(11)Zhang, J.; Kamstra, J. H.; Ghorbanzadeh, M.; Weiss, J. M.; Hamers, T.;Andersson, P. L. In Silico Approach To Identify Potential Thyroid Hormone Disruptors among Currently Known Dust Contaminants and Their Metabolites. Environ. Sci. Technol. 2015, 49, (16), 10099-10107.

(12)Marchesini, G. R.; Meimaridou, A.; Haasnoot, W.; Meulenberg, E.; Albertus, F.; Mizuguchi, M.; Takeuchi, M.; Irth, H.;Murk, A. J. Biosensor discovery of thyroxine transport disrupting chemicals. Toxicol. Appl. Pharmacol. 2008, 232, (1), 150-160.

(13)Meerts, I. A. T. M.; van Zanden, J. J.; Luijks, E. A. C.; van Leeuwen-Bol, I.; Marsh, G.; Jakobsson, E.; Bergman, Å.;Brouwer, A. Potent Competitive Interactions of Some Brominated Flame Retardants and Related Compounds with Human Transthyretin in Vitro. Toxicol. Sci. 2000, 56, (1), 95-104.

(14)Morgado, I.; Hamers, T.; Van der Ven, L.;Power, D. M. Disruption of thyroid hormone binding to sea bream recombinant transthyretin by ioxinyl and polybrominated diphenyl ethers. Chemosphere 2007, 69, (1), 155-163.

(15)Zhang, J.; Begum, A.; Brannstrom, K.; Grundstrom, C.; Iakovleva, I.; Olofsson, A.; Sauer-Eriksson, A. E.;Andersson, P. L. Structure-Based Virtual Screening Protocol for in Silico Identification of Potential Thyroid Disrupting Chemicals Targeting Transthyretin. Environ. Sci. Technol. 2016, 50, (21), 11984-11993.

(16)Cheek, A. O.; Kow, K.; Chen, J.;McLachlan, J. A. Potential mechanisms of thyroid disruption in humans: interaction of organochlorine compounds with thyroid receptor, transthyretin, and thyroid-binding globulin. Environ. Health Persp. 1999, $107,(4), 273-278$.

(17)van den Berg, K. J. Interaction of chlorinated phenols with thyroxine binding sites of human transthyretin, albumin and thyroid binding globulin. Chem-Biol. 
Interact. 1990, 76, (1), 63-75.

(18)Simon, E.; van Velzen, M.; Brandsma, S. H.; Lie, E.; Loken, K.; de Boer, J.; Bytingsvik, J.; Jenssen, B. M.; Aars, J.; Hamers, T.;Lamoree, M. H. Effect-directed analysis to explore the polar bear exposome: identification of thyroid hormone disrupting compounds in plasma. Environ. Sci. Technol. 2013, 47, (15), 8902-8912.

(19)Marchesini, G. R.; Meulenberg, E.; Haasnoot, W.; Mizuguchi, M.;Irth, H. Biosensor Recognition of Thyroid-Disrupting Chemicals Using Transport Proteins. Anal. Chem. 2006, 78, (4), 1107-1114.

(20)Simon, E.; Bytingsvik, J.; Jonker, W.; Leonards, P. E.; de Boer, J.; Jenssen, B. M.; Lie, E.; Aars, J.; Hamers, T.;Lamoree, M. H. Blood plasma sample preparation method for the assessment of thyroid hormone-disrupting potency in effect-directed analysis. Environ. Sci. Technol. 2011, 45, (18), 7936-7944.

(21)Hamers, T.; Kamstra, J. H.; Sonneveld, E.; Murk, A. J.; Kester, M. H.; Andersson, P. L.; Legler, J.;Brouwer, A. In vitro profiling of the endocrine-disrupting potency of brominated flame retardants. Toxicol. Sci. 2006, 92, (1), 157-173.

(22)Cao, J.; Lin, Y.; Guo, L. H.; Zhang, A. Q.; Wei, Y.;Yang, Y. Structure-based investigation on the binding interaction of hydroxylated polybrominated diphenyl ethers with thyroxine transport proteins. Toxicology 2010, 277, (1-3), 20-28.

(23)Dufour, P.; Pirard, C.;Charlier, C. Validation of a novel and rapid method for the simultaneous determination of some phenolic organohalogens in human serum by GC-MS. J. Chromatogr. B 2016, 1036-1037, 66-75.

(24)Chu, S.;Letcher, R. J. Halogenated phenolic compound determination in plasma and serum by solid phase extraction, dansylation derivatization and liquid chromatography-positive electrospray ionization-tandem quadrupole mass spectrometry. J. Chromatogr. A 2013, 1320, 111-117.

(25)Petropoulou, S.-S. E.; Duong, W.; Petreas, M.; Park, J.-S. Fast liquid chromatographic-tandem mass spectrometric method using mixed-mode phase chromatography and solid phase extraction for the determination of 12 mono-hydroxylated brominated diphenyl ethers in human serum. J. Chromatogr. A 
2014, 1356, 138-147. 